\title{
Detection of a novel PAX6 variant in a Chinese family with multiple ocular abnormalities
}

\author{
Junyi Ouyang ${ }^{1,2,3}$, Ziyan Cai ${ }^{4}$, Yinjie Guo ${ }^{4}$, Fen $\mathrm{Nie}^{4}$, Mengdan $\mathrm{CaO}^{4}$ and Xuanchu Duan ${ }^{1,2,3^{*}}$
}

\begin{abstract}
Background: Aniridia is a congenital, panocular disease that can affect the cornea, anterior chamber angle, iris, lens, retina and optic nerve. PAX6 loss-of-function variants are the most common cause of aniridia, and variants throughout the gene have been linked to a range of ophthalmic abnormalities. Furthermore, particular variants at a given site in PAX6 lead to distinct phenotypes. This study aimed to characterize genetic variants associated with congenital aniridia in a Chinese family.
\end{abstract}

Methods: The proband and family underwent ophthalmologic examinations. DNA was sampled from the peripheral blood of all 6 individuals, and whole-exome sequencing was performed. Sanger sequencing was used to verify the variant in this family members.

Results: A novel variant (c.114_119delinsAATTTCC: p.Pro39llefsTer17) in the PAX6 gene was identified in subjects II-1, III-1 and III-2, who exhibited complete aniridia and cataracts. The proband and the proband's brother also had glaucoma, high myopia, and foveal hypoplasia.

Conclusions: We identified that a novel PAX6 frameshift heterozygous deletion variant is the predominant cause of aniridia in this Chinese family.

Trial registration: We did not perform any health-related interventions for the participants.

Keywords: PAX6, Aniridia, DNA variant, Phenotype

\section{Background}

Aniridia is an eye disorder defined as partial or complete absence of the iris that can be congenital or caused by injury. Congenital aniridia is a sporadic [1] rare condition that affects 1:64,000-1:96,000 individuals, and up to twothirds of patients exhibit an autosomal dominant form of the disorder [2].

Although the absence of the iris is the most prominent sign of this condition, congenital aniridia is also

*Correspondence: duanxchu@126.com

3 Aier Glaucoma Research Institute, Changsha Aier Eye Hospital, Changsha, Hunan, China

Full list of author information is available at the end of the article associated with abnormalities in the cornea, retina, lens, anterior chamber angle, and optic nerve. Most aniridia patients exhibit macular hypoplasia, nystagmus, and significant visual impairment, and a small subset have optic nerve hypoplasia [3]. In addition, patients with aniridia often develop a range of secondary ocular complications, including cataracts, aniridic keratopathy, and glaucoma. In fact, glaucoma affects up to $70 \%$ of aniridic patients [4].

Over 500 variants in the $P A X 6$ gene and its regulatory regions have been characterized to date. Many of these variants account for PAX6 haploinsufficiency, which leads to significant ocular and systemic abnormalities [5]. original author(s) and the source, provide a link to the Creative Commons licence, and indicate if changes were made. The images or other third party material in this article are included in the article's Creative Commons licence, unless indicated otherwise in a credit line to the material. If material is not included in the article's Creative Commons licence and your intended use is not permitted by statutory regulation or exceeds the permitted use, you will need to obtain permission directly from the copyright holder. To view a copy of this licence, visit http://creativecommons.org/licenses/by/4.0/. The Creative Commons Public Domain Dedication waiver (http://creativeco mmons.org/publicdomain/zero/1.0/) applies to the data made available in this article, unless otherwise stated in a credit line to the data. 
In the present study, we describe a novel PAX6 variant associated with congenital aniridia in a Chinese family.

\section{Methods}

\section{Subjects and Clinical evaluation}

Thorough ophthalmologic examinations were performed for the proband and her brother, including tests of visual acuity, intraocular pressure (IOP), slit-lamp analyses, anterior segment photography, visual field tests (Humphrey 750,Carl Zeiss, Germany), funduscopy, ultrasonic B analyses (Chiescan Quantel Medical, France), gonioscopic analyses, OCTA (optical coherence tomography angiography) assessments (RTVue-XR Avanti, v2017.1.0; OptoVue, Inc., CA, USA), and ultrasound biomicroscope (UBM) assessments (SW China). The proband's other family members underwent a simple slit lamp examination.

\section{Variant screening and sequence analysis Genomic DNA extraction}

Approximately $4 \mathrm{ml}$ of peripheral blood was sampled from the proband and her brother. Genomic DNA was extracted using a genomic DNA extraction and purification kit (TIANamp Blood DNA kit, \#DP348-03) following the manufacturer's protocol. The genomic DNA samples were stored at $-20^{\circ} \mathrm{C}$ until use.

\section{Library construction}

1. Genome-wide library construction: DNA enzymatic fragmentation and genome-wide library construction were carried out using the DNA library construction kit of YEASEN Biology Company (Hieff NGS ${ }^{\circledR}$ OnePot DNA Library Prep Kit for Illumina ${ }^{\circledR}$, YEASEN).

2. Construction of the clinical whole-exome capture library: a XGen Exome Research Panel V1.0 (Integrated DNA Technologies, Inc., USA) of the IDT company was used for capture and to construct the library of the proband and brother.

\section{Clinical whole-exome sequencing}

Paired-end sequencing was performed using the Illumina (San Diego, ca) sequencing platform with PE 150 patterns.

\section{Bioinformatics analysis}

Raw reads of low quality were removed, and the remaining reads were mapped to the UCSC (University of California Santa Cruz) hg19 reference genome (http:// genome.ucsc.edu/). Single-nucleotide variations (SNVs) and insertion-deletion (InDel) variants were detected using the HaplotypeCaller function of Genome Analysis
ToolKit (GATK, http://software.broadinstitute.org/gatk/). These annotated variants were then filtered based on the Annovar (http://www.openbioinformatics.org/annovar/) database. The databases used for pathogenicity prediction were SIFT (http://sift.jcvi.org), Polyphen2_HDIV (http:// genetics.bwh.harvard.edu/pph2), Polyphen2_HVAR (http://genetics.bwh.harvard.edu/pph2), LRT (http:// www.genetics.wustl.edu/jflab/lrt_query.html), variantTaster (http://www.varianttaster.org/), variantAssessor (http://variantassessor.org/r3/), FATHMM (http:// fathmm.biocompute.org.uk), PROVEAN (http://provean. jcvi.org/index.php), MetaSVM (https://omictools.com/ meta-svmtool), MetaLR (http://www.ensembl.info/tag/ metalr/), M-CAP (http://bejerano.stanford.edu/mcap/), fathmm-MKL_coding (http://fathmm.biocompute.org. uk/fathmmMKL.htm). Quality control requirements: data volume $>=6 \mathrm{~GB}$, average coverage $>=150 \mathrm{X}, 30 \mathrm{X}$ coverage $>=98.5 \%$, Q30 Qualification rate $(\%)>=89.17 \%)$.

\section{Variant pathogenicity analysis}

The guidelines of the American College of Medical Genetics and Genomics (ACMG) were used to facilitate appropriate data analysis (Table 3 ). Only genetic variations with known, definitive genetic associations were analysed. Genes with unknown pathogenicity or functionality were omitted from these analyses. In addition, common benign polymorphic variants, synonymous variants, and intronic variants not altering mRNA splicing were not included unless they have previously been reported in the literature as being pathogenic or were present in the database.

\section{Variant verification}

Detected variations were validated by Sanger sequencing in the Chinese family. Primer3Plus (http://www.prime r3plus.com/cgi-bin/dev/primer3plus.cgi) was used to design primers for PAX6 gene c.114_119delinsAATTTC C: p.Pro39llefsTer17, and in-silico PCR (http://genome. ucsc.edu/cgi-bin/hgPcr) was used to verify primer specificity (Table 1). PCR amplification products for family members were sequenced using an ABI3730s AUTOMATIC DNA sequence Analyser (3730 DNA Analyser), and the results were analysed and compared using CodonCode Aligner software (CodonCode Corporation, USA) (Fig. 1).

\section{Results}

Clinical data

\section{The proband}

A 13-year-old girl presented to our hospital complaining of bilateral blurred vision with no history of surgery or medical treatment of either eye. Her IOPs were $44 \mathrm{mmHg}$ OD and $38 \mathrm{mmHg}$ OS, as detected by Goldmann 
Table 1 Sequencing primer details

\begin{tabular}{|c|c|c|c|}
\hline Primer Name & Sequence & Amplified fragment length (bp) & Amplification reaction conditions. \\
\hline SG2560_F & $\begin{array}{l}5^{\prime}-\mathrm{TACAGTAAGAAA} \\
\text { TGAAGAGAGGGC } \\
\text { GTT }-3^{\prime}\end{array}$ & 499 & $\begin{array}{l}3 \mathrm{~min} \text { at } 95^{\circ} \mathrm{C} \text { for PRE-denaturation, } 30 \mathrm{~s} \text { at } 95^{\circ} \mathrm{C} \text { for denatura- } \\
\text { tion, } 30 \mathrm{~s} \text { at } 60^{\circ} \mathrm{C} \text { for primer annealing, } 40 \mathrm{~s} \text { at } 72^{\circ} \mathrm{C} \text { for primer } \\
\text { extension, }\end{array}$ \\
\hline SG 2560_R & $\begin{array}{l}5^{\prime}-\text { GGGCACGGTTGC } \\
\text { TTGGACT - 3' }\end{array}$ & & 30 cycles,and another $5 \mathrm{~min}$ at $72^{\circ} \mathrm{C}$ for primer extension. \\
\hline
\end{tabular}

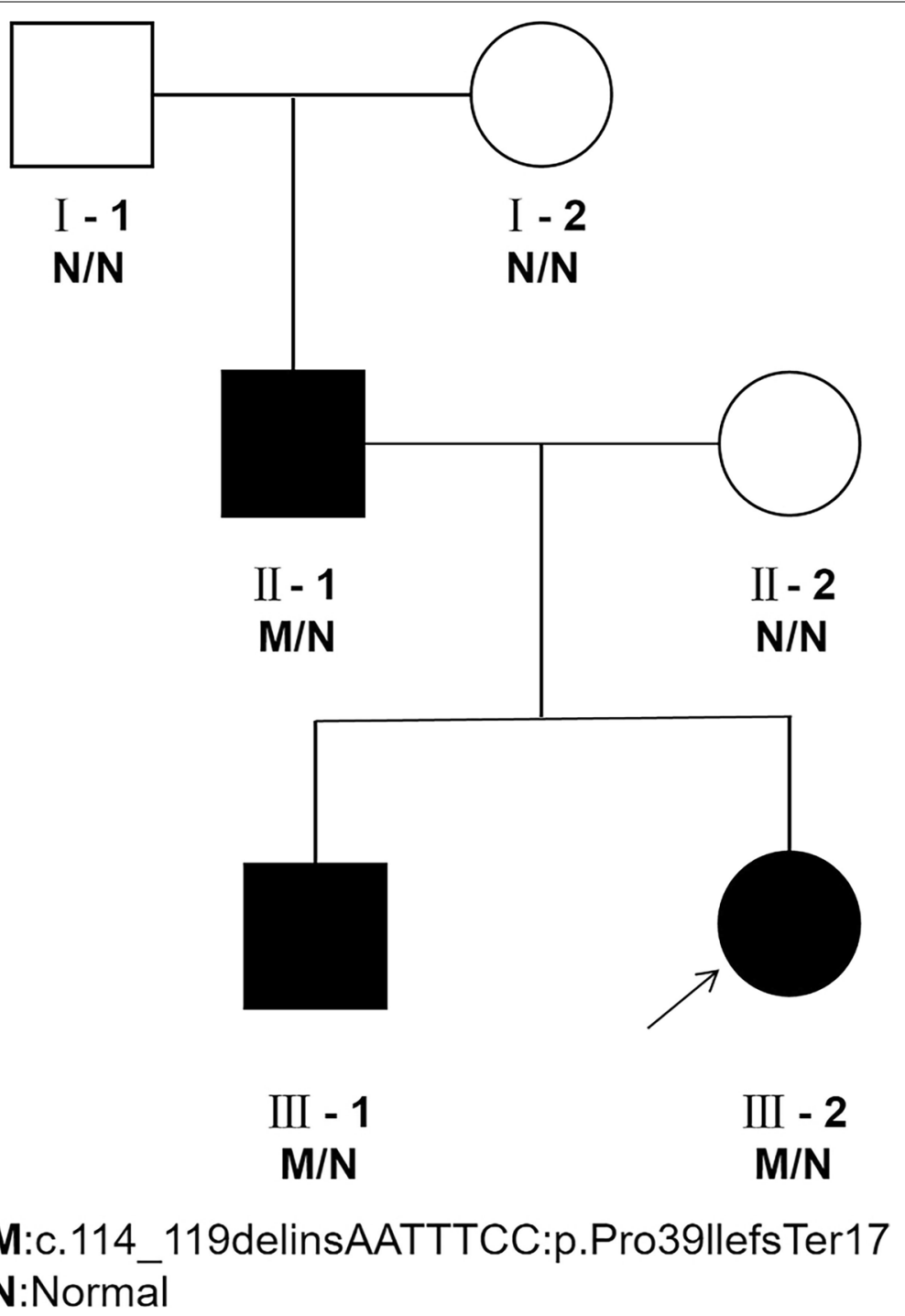

Fig. 1 Pedigree of a Chinese family with aniridia. Squares and circles correspond to males and females, respectively. Black and white shapes correspond to affected and unaffected individuals, respectively. The proband is indicated with an arrow

tonometry; her best-corrected visual acuity (BCVA) was 20/100 OD and 20/125 OS. Refractive errors were -8.5 D OD and-6.0 D OS, with eyeball axis length (AL) values of $26.3 \mathrm{~mm}$ OD and $26.0 \mathrm{~mm}$ OS. The anterior chamber in both eyes appeared normal, and peripheral angles in both eyes were open. A slit-lamp examination showed the presence of bilateral peripheral cataracts and posterior capsular opacification, and UBM examination revealed iris coloboma. Further fundus examination revealed large optic disc with bilateral glaucomatous cupping 
and peripapillary atrophy. OCTA examination indicated diffuse superior and inferior RNFL (retinal nerve fibre layer) thinning, reduced wiVD (whole-image vessel density), idVD (inside disc vessel density), ppVD (peripapillary vessel density) vessel density, and significant foveal hypoplasia (Table 2). Visual field tests highlighted bilateral glaucomatous defects (Fig. 2). The proband had no other discomfort, particularly hearing loss or abnormal olfaction.

\section{The brother of the proband}

The 23-year-old brother of the proband reported a history of glaucoma that had been diagnosed one year prior in a different hospital, and he had been using IOP-lowering eye drops since.

His BCVA in both eyes was 20/80, with refractive error values of $-9.5 \mathrm{D}$ OD and-10.25 D OS; eyeball $\mathrm{AL}$ values were $26.7 \mathrm{~mm}$ OD and $26.5 \mathrm{~mm}$ OS. He exhibited many of the same ophthalmic abnormalities as his sister, including complete aniridia, cataracts, glaucoma, high myopia, and foveal hypoplasia. In addition, the brother showed a decreased VD compared to the usual observation for healthy eyes. The superior and inferior RNFL of the brother's eyes were thicker than that of his sister's eyes (Table 2), and he had fewer pronounced bilateral glaucomatous visual field defects (Fig. 3). The proband's brother also had no hearing loss or abnormal olfaction.

\section{Other family members}

The proband's father had limited vision, such as light perception, and a simple examination showed severe cataracts and complete aniridia. The proband's mother and grandparents had no obvious eye problems.

\section{Variant analysis}

Next-generation sequencing analyses demonstrated the presence of a heterozygous frameshift deletion variant (c.114_119delinsAATTTCC: p.Pro39llefsTer17) in exon 5 of the PAX6 gene. This variant, consisting of a 6-bp deletion and a 7-bp insertion, results in a frameshift from the 39th proline codon, resulting in the generation of a premature stop codon (Fig. 4). Based ACMG, the variant is a pathogenic variant (Table 3).

\section{Discussion}

By analysing a Chinese family with a history of congenital aniridia, we identified a novel hybrid variant (c.114_119delinsAATTTCC: p.Pro39llefsTer17) in the PAX6 gene. This variant comprises a 6-bp deletion and a 7-bp insertion resulting in premature truncation of the PAX6 protein. The affected brother and sister exhibited shared ophthalmic abnormalities, including cataracts, nystagmus, glaucoma, aniridia, and macular fovea hypoplasia. The PAX6 gene, located on chromosome 11p13, was first characterized by Ton et al. in 1991 [6]. PAX6 encodes a transcriptional regulator that is important for the development of organs and tissues, including the eyes. PAX6 expression is detectable in the iris, lens, optic disc, corneal epithelium, ciliary body, retinal neuroepithelium, and retinal pigment epithelium. In 2005, Tzoulaki et al. characterized human PAX6 variants and found that variants throughout the gene were associated with aniridia and related phenotypes [7]. In a study of 95 Chinese patients with aniridia, You et al. found PAX6 loss-of-function variants to be the most common cause of aniridia [8]. The PAX6 variant identified in these siblings in the present study (c.114_119delinsAATTTC C: p.Pro39llefsTer17) causes a frameshift from the 39th codon, leading to a premature stop codon. In light of prior studies, we hypothesize that this variant is likely to be the primary cause of the aniridia and other observed ophthalmic abnormalities in these siblings.

In their prior study of 95 Chinese aniridia patients, You et al. identified 47 different variants associated with the aniridia phenotype, including 6 frameshift InDel variants, 12 nonsense variants, 2 missense variants, 1 run-on variant, 1 synonymous variant, and 15 variants that altered mRNA splicing [8]. The human gene variant database (HGMD) currently includes 479 pathogenic PAX6 variants (http://www.hgmd.cf.ac.uk/ac/gene.php? gene=PAX6). In total, 20 reports to date have described cases of patients with both insertion and deletion variants in PAX6, and such combination variants are likely to be associated with serious ophthalmic abnormalities. Our observations of abnormalities, including aniridia and glaucoma, in the patients in the present study are thus consistent with these prior studies.

Table 2 RNFL, wiVD (whole-image vessel density), idVD (inside disc vessel density), and ppVD (peripapillary vessel density) values for the proband and the proband's brother

\begin{tabular}{llllllll}
\hline Patient & RNFL $(\boldsymbol{\mu m})$ & & wiVD & & idVD & & \multicolumn{2}{c}{ PPVD\% } \\
\hline eye & OD & OS & OD & OS & OD & OS & OD \\
proband & 78 & 80 & 43.6 & 39.5 & 32.8 & 40.2 & 41.7 \\
proband's brother & 85 & 82 & 42.3 & 40.6 & 43.8 & 33.4 & 43.4 \\
\hline
\end{tabular}



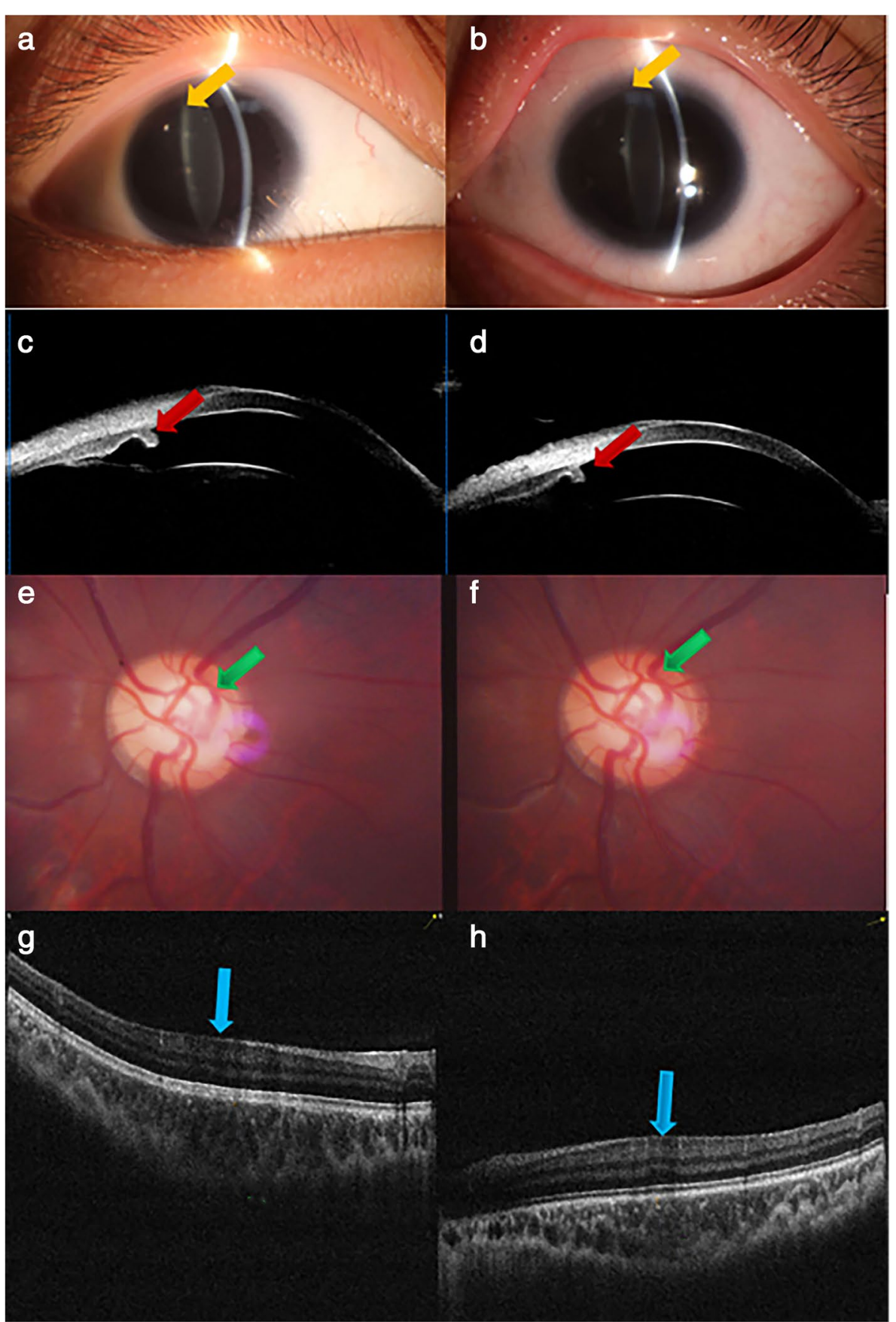

Fig. 2 Clinical findings for the proband. The right and left columns correspond to the left and right eyes, respectively. Images of the anterior segment $(\mathbf{a}, \mathbf{b})$ and horizontally scanned UBM images of the anterior chamber $(\mathbf{c}, \mathbf{d})$. Images of the optic disc $(\mathbf{e}, \mathbf{f})$ and macular OCT examination $(\mathbf{g}, \mathbf{h})$. The yellow and red arrows indicated the residual image of iris. The blue arrows indicated the structure of fovea of macula. The green arrow indicated the structure of optic disc

The PAX6 protein is composed of four domains: two DNA-binding domains, including an N-terminal 128amino acid paired box domain (PD) and a 61-amino acid homeodomain (HD), as well as a 79-amino acid glycine-rich hinge region and a $\mathrm{C}$-terminal proline-rich serine transactivation domain $[9,10]$. variants throughout PAX6 have been linked to a range of ophthalmic abnormalities, with particular variants at a given site leading to distinct phenotypes. In the present subjects, glaucoma manifested at an earlier age and was more 

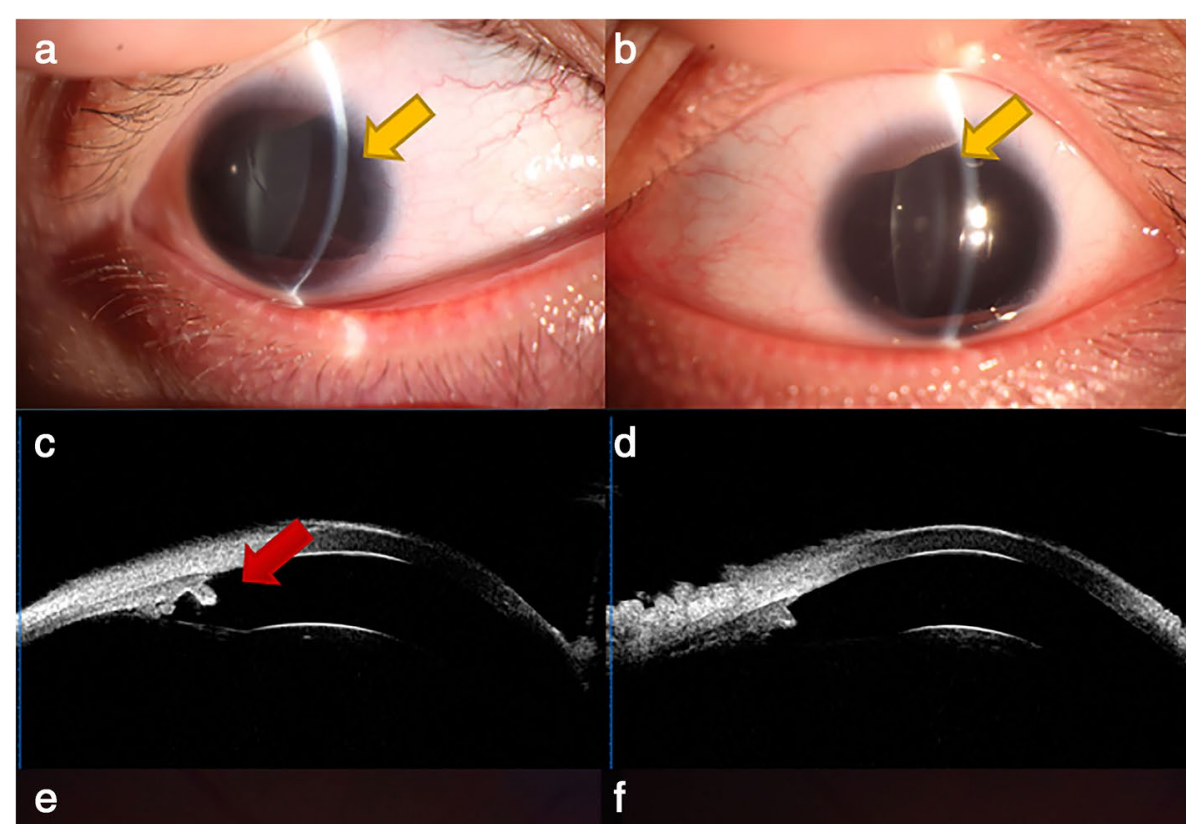

e
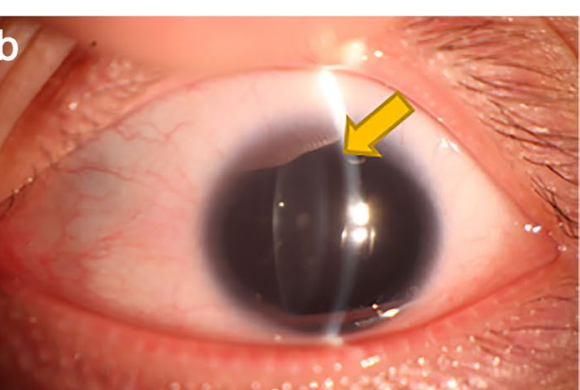
a

\section{$\begin{array}{lllll}24251 & 31824261 & 31824271 & 31824281 & 31824291\end{array}$ 'GCAGAATTCGGGAAATGTCGCACGGCCGGGCCCCGCTGTGAGCTAGC:}

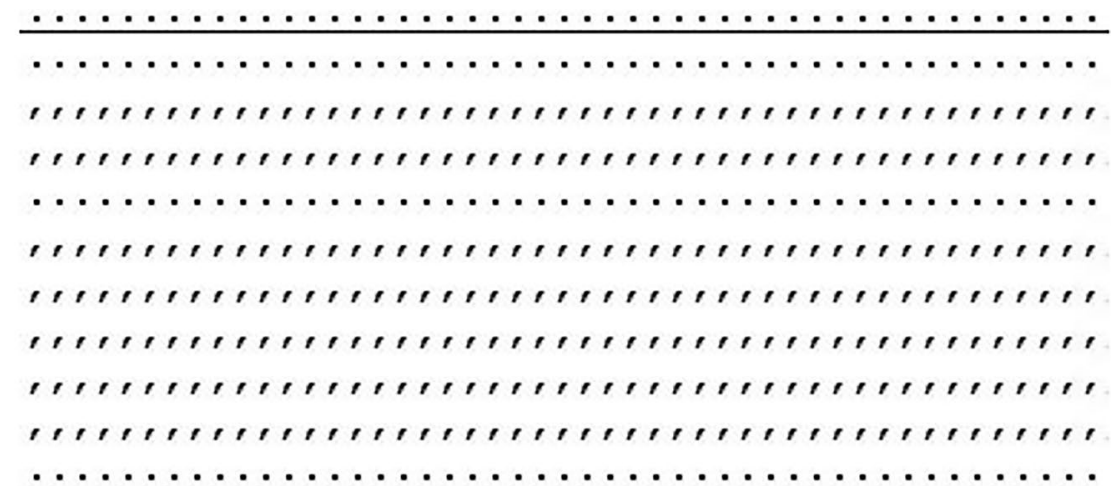

b
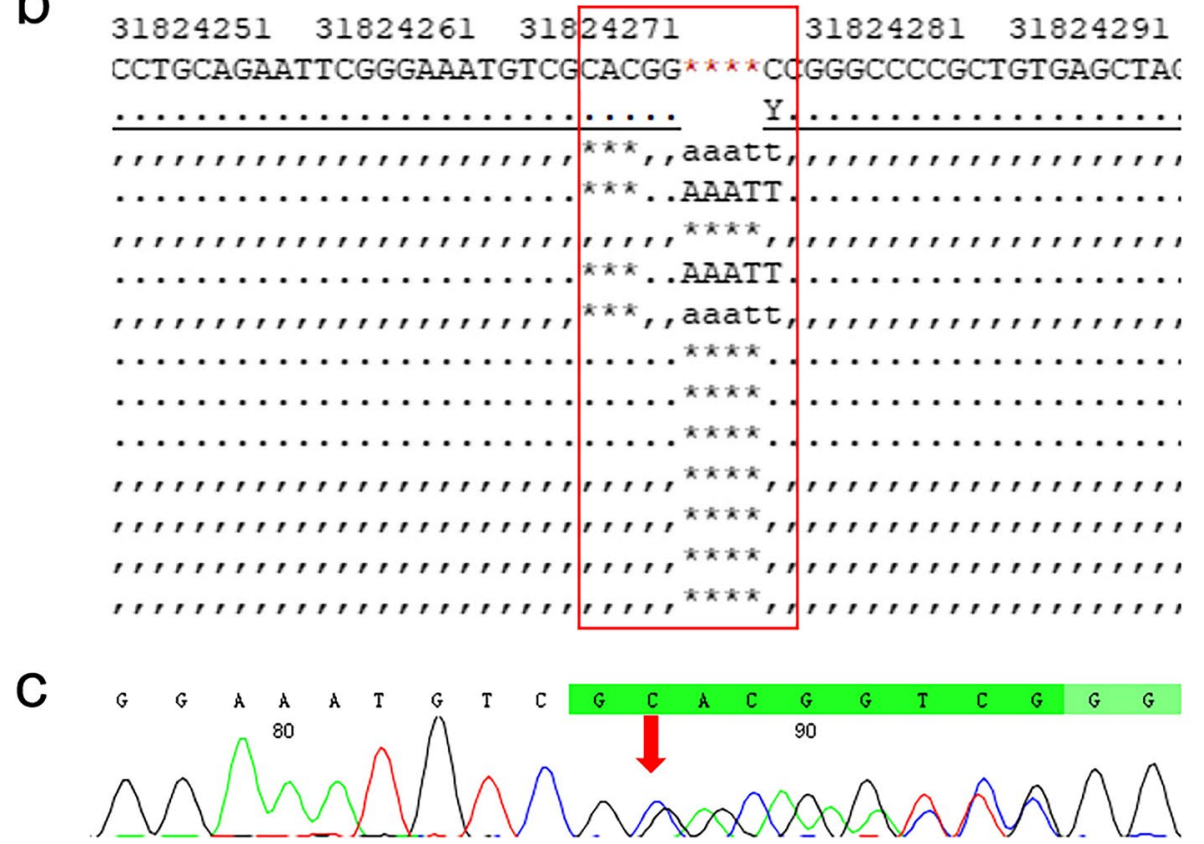

Fig. 4 Sequence chromatograms showing the wild-type (a) and mutated (b) PAX6 gene. The red box demonstrates the presence of a heterozygous frameshift deletion variant (c.114_119delinsAATTTCC: p.Pro39llefsTer17) in exon 5 of the PAX6 gene. The red arrows indicate the site of the variant, with the dual peaks corresponding to the presence of the indicated heterozygous insertion variants

Table 3 The classification refers to ACMG guidelines

\begin{tabular}{|c|c|c|}
\hline ACMG & Description of evidence & Classification results \\
\hline PVS1 & $\begin{array}{l}\text { The pax6 gene in the clingen database (https://clinicalgenome.org/) was recorded with a single dose } \\
\text { sensitive gene with a score of } 3 \text {. }\end{array}$ & Pathogenic variation \\
\hline PM2_Supporting & $\begin{array}{l}\text { It is a rare variant, not included in the Genome Aggregation Database (gnomAD,https://gnomad.broad } \\
\text { institute.org/about) East Asian database. }\end{array}$ & \\
\hline PP4 & The patient's clinical symptoms and family history were anastomotic with PAX6 gene abnormality. & \\
\hline
\end{tabular}


frame lead to premature protein truncation as a result of nonsense-mediated mRNA decay (NMD). In such a dose-effect model, a single wild-type allele of PAX6 is insufficient to ensure normal ocular development, leading to ophthalmic abnormalities [12]. Subtle phenotypic differences between patients with various PAX6 variants may thus be attributable to slight differences in intracellular PAX6 levels [13]. In the present study, we identified a novel heterozygous frameshift variant in PAX6 that results in a frameshift from the 39th proline codon and the generation of a premature stop codon. This variant begins in exon 5 in the PD domain and leads to truncation of the LNK (linker, glycine-rich hinge region), HD, and PST domains of the protein, resulting in a shortened polypeptide that is unlikely to be functional [12]. Haploinsufficiency is likely to explain the observed aniridia phenotype in the subjects of the present study, though the mechanistic link between genotype and phenotype in these patients needs to be fully characterized in future studies.

\section{Conclusions}

In summary, we identified the novel heterozygous c.114_119delinsAATTTCC: p.Pro39llefsTer17 variant of the PAX6 gene as a putative cause of aniridia in a Chinese family. These results expand the spectrum of known variants that cause PAX6-triggered congenital aniridia and enhance our understanding of the genetic aetiology of this condition. Furthermore, our findings may aid in the genetic diagnosis of aniridia.

\begin{abstract}
Abbreviations
IOP: Intraocular pressure; OCTA: Optical coherence tomography angiography; UBM: Ultrasound biomicroscope; SNVs: Single nucleotide variations; InDel: Insertion-deletion; GATK: Genome Analysis ToolKit; ACMG: The guidelines of the American College of Medical Genetics and Genomics; BCVA: Bestcorrected visual acuity; RNFL: Retinal nerve fibre layer) thinning; wiVD: Whole image vessel density; idVD: Inside disc vessel density; ppVD: Peripapillary vessel density; VD: Vessel density; HGMD: The human gene variant database; PD: Paired box domain; HD: Homeodomain; NMD: Nonsense-mediated mRNA decay; PTCs: Premature termination codon.
\end{abstract}

\section{Supplementary Information}

The online version contains supplementary material available at https://doi. org/10.1186/s12886-022-02256-7.

Additional file 1.

\section{Acknowledgements}

I am greatly indebted to my supervisor, Professor Xuanchu Duan, for his valuable instructions and suggestions on my thesis as well as his careful reading of the manuscript. I also owe much to my friends and classmates for their valuable suggestions and critiques, which were of help and importance in making the thesis a reality. Last, I would like to thank my parents and husband for their loving considerations and great confidence in me throughout the years.

\section{Authors' contributions}

Junyi Ouyang involved in the conception, design of study and wrote the article. Ziyan Cai analyzed, interpreted the data and critical revised the article. Yinjie Guo analyzed, interpreted the data. Fen Nie analyzed, interpreted the data. Mengdan Cao analyzed, interpreted the data. Xuanchu Duan involved in the conception, design of study and critical revised the article. All authors have read and approved the manuscript.

\section{Funding}

This work was supported by the National Natural Science Foundation of China (Grant No. 81670859, 81970801 to XD), Science and Technology Foundation of Changsha, Hunan, China (Grant No. kh1801229 to XD), Natural Science Foundation of Hunan Province, China (Grant No. $2019 \mathrm{JJ} 40001$ to XD) and Science and Technology Foundation of Aier Eye Hospital Group, China (Grant No. AR1 906D1, AM1906D2 to XD and Aier Glaucoma Research Institute). The funding provided support for the collection and analysis of data.

\section{Availability of data and materials}

All data generated or analysed during this study are included in this published article [and its supplementary information files].

\section{Declarations}

\section{Ethics approval and consent to participate}

This study was performed in accordance with the Declaration of Helsinki and approved by the ethics committee of the Changsha Aier Eye Hospital, China (No:2019KYPJ001). Written informed consent was obtained from all enrolled patients.

\section{Consent for publication}

We confirm that the manuscript has been read and approved by all named authors and that there are no other persons who satisfied the criteria for authorship but are not listed. We further confirm that the order of authors listed in the manuscript has been approved by all of us. The brother and the father of the proband, who were not minors, gave written consent for their personal or clinical details along with any identifying images to be published in this study.

\section{Competing interests}

The authors declare that they have no competing interests.

\section{Author details}

'Jinan University, Guangzhou, Guangdong, China. ${ }^{2}$ Aier School of Ophthalmology, Central South University, Changsha, Hunan, China. ${ }^{3}$ Aier Glaucoma Research Institute, Changsha Aier Eye Hospital, Changsha, Hunan, China. ${ }^{4}$ Department of Ophthalmology, The Second Xiangya Hospital, Central South University, Changsha, Hunan Province, China.

Received: 11 May 2021 Accepted: 5 January 2022

Published online: 16 January 2022

\section{References}

1. Koushik Aniridia [M]. Treasure Island: StatPearls Publishing; 2020. https:// www.ncbi.nlm.nih.gov/books/NBK538133/.

2. Xiao Y, Liu XQ, Yang $C$, et al. PAX6 a novel heterozygous variant found in a Chinese family with congenital Aniridia. Genet Test Mol Biomarkers. 2019;23:495-500. https://doi.org/10.1089/gtmb.2018.0334.

3. Glaser T, Jepeal L, Edwards JG, et al. PAX6 gene dosage effect in a family with congenital cataracts, aniridia, anophthalmia and central nervous system defects. Nat. Genet. 1994;7:463-71. https://doi.org/10.1038/ ng0894-463.

4. Eugen G, Constantin R, Gwendolyn G. Glaucoma and frequency of ocular and general diseases in 30 patients with aniridia:a clinical study. Eur J Ophthalmol. 2012;22:104-10. https://doi.org/10.5301/EJO.2011.8318.

5. Dulce LC, Gavin A, Marta C, et al. PAX6 the Spectrum of variants and genotype-phenotype correlations in the eye. Genes (Basel). 2019;10: undefined. https://doi.org/10.3390/genes10121050. 
6. Ton $\mathrm{CC}$, Hirvonen $\mathrm{H}$, Miwa $\mathrm{H}$, et al. Positional cloning and characterization of a paired box-and homeobox-containing gene from the aniridia region. Cell. 1991;67:1059-74. https://doi.org/10.1016/0092-8674(91)90284-6.

7. Ioanna T, White lan MS, Hanson Isabel M. PAX6 variants:genotypephenotype correlations. BMC Genet. 2005;6:27. https://doi.org/10.1186/ 1471-2156-6-27.

8. You B, Zhang XH, Xu K, et al. PAX6variant spectrum of and clinical findings in 95 Chinese patients with aniridia. Mol.Vis. 2020;26:226-34 http://www. molvis.org/molvis/v26/226

9. Tang HK, Singh S, Saunders GF. Dissection of the transactivation function of the transcription factor encoded by the eye developmental gene PAX6. J Biol Chem. 1998;273:7210-21. https://doi.org/10.1074/jbc.273.13. 7210.

10. Treisman J, Harris E, Desplan C. The paired box encodes a second DNA-binding domain in the paired homeo domain protein. Genes Dev. 1991;5(4):594-604. https://doi.org/10.1101/gad.5.4.594.

11. Singh S, Tang HK, Lee JY, et al. Truncation variants in the transactivation region of PAX6 result in dominant-negative mutants. J.Biol.Chem. 1998;273:21531-41. https://doi.org/10.1074/jbc.273.34.21531.

12. Smith JE, Baker KE. Nonsense-mediated RNA decay — a switch and dial for regulating gene expression. Bioessays. 2015;37(6):612-23. https://doi.org/ 10.1002/bies.201500007.

13. Marie-Claire V, Anne-Laure P, David O, et al. Screening for PAX6 gene variants is consistent with haploinsufficiency as the main mechanism leading to various ocular defects. Eur.J.Hum.Genet. 2003;11:163-9. https://doi. org/10.1038/sj.ejhg.5200940.

\section{Publisher's Note}

Springer Nature remains neutral with regard to jurisdictional claims in published maps and institutional affiliations.

- fast, convenient online submission

- thorough peer review by experienced researchers in your field

- rapid publication on acceptance

- support for research data, including large and complex data types

- gold Open Access which fosters wider collaboration and increased citations

- maximum visibility for your research: over $100 \mathrm{M}$ website views per year

At BMC, research is always in progress.

Learn more biomedcentral.com/submissions 